\title{
Structural Heterogeneity of an Amorphous-Nanocrystalline Alloy Fe77Cu1Si16B6 in the Nanometer Range
}

\author{
Frolov A.M., Ansovich A.V., Kraynova G.S., TKacheV V. V., DolzhiKov, \\ S.V., Plotnikov V.S., Ralin A.Yu., Fedorets A. N. \\ Far Eastern Federal University \\ FEFU Campus, 10 Ajax Bay, Russky Island, Vladivostok, 690922, \\ RUSSIAN FEDERATION
}

\begin{abstract}
In this article, an alloy of the Finemet type $\mathrm{Fe}_{77} \mathrm{Cu}_{1} \mathrm{Si}_{16} \mathrm{~B}_{6}$ obtained by quenching from a liquid state (spinning method) in the initial state is investigated. The main research methods were scanning and transmission electron microscopy. Methods for describing multiscale structural heterogeneities in amorphous-nanocrystalline alloys have been developed, allowing the structural state to be described and its influence on the physicochemical and technical properties to be determined depending on the technological conditions for obtaining these alloys. Representation of electron microscopic images in the form of Fourier spectra made it possible to reveal the nature of the formation of short- and middle-order in amorphous-nanocrystalline alloys according to the principle of selfsimilar spatial structures. The analysis of electron microscopic images by integral Lebesgue measures revealed density fluctuations over the alloy volume, which corresponds to the hierarchical representation of structural inhomogeneities in amorphous metallic alloys.
\end{abstract}

Keywords: amorphous nanocrystalline alloys, Fourier analysis, anisotropy, heterogeneity, density fluctuations.

Received: April 2, 2021. Revised: November 2, 2021. Accepted: December 13, 2021. Published: January 5, 2022.

\section{Introduction}

$\mathrm{M}$ odern scientific and technological progress is accompanied, on the one hand, by the creation of fundamentally new technologies and processes, on the other requires the use of materials that are more relevant today. The latest technologies make it possible to improve already known materials or create new ones.

Partially crystalline alloys (Finemet) are a new class of materials obtained by rapid quenching from a liquid state. Moreover, in terms of physical and mechanical properties, such two-phase systems are superior to the properties of both nanocrystalline and amorphous materials, thereby creating a noticeable synergistic effect [1-9]. Amorphous metallic alloys present short ( 0.2 to $0.5 \mathrm{~nm}$.) and medium range order ( 0.5 to $2 \mathrm{~nm}$.), it is confirmed by various experimental methods, including transmission electron microscopy (TEM) $[3,10-$ 12].High-resolution TEM (HRTEM) makes it possible to determine the levels of inhomogeneities of different subnanometer spatial scales in amorphous metallic alloys obtained by rapid quenching from the melt. The crystalline phase has an ordered atomic structure, the atomic structure of the amorphous phaseis devoid of translational symmetry and has only pronounced topological and compositional shortrange orders. Consequently amorphous-nanocrystalline materials can be considered natural composites, which have important properties for practical use [13-19]. Such materials have a pronounced hierarchical structure of various spatial scales of inhomogeneity, which provides their special physical properties during the applications [5].

Due to their physical properties, Fe-based alloys have become the most interesting for practical applications (in magnetic recording heads, transformers, electronic devices, etc.) $[20,21]$. Knowledge of the correlation of the structure, as well as the mechanism of its formation, gives a clear understanding of the known properties of materials, including Finemet, that will allow them to be improved and also to obtain new ones with given characteristics and devices based on them. Thus, the issues related to the study of the structure of alloys are extremely topical [1-3,13-16].

The most informative group of methods for studying the structure is electron microscopy. Electron microscopy supplemented by analytical methods of statistical analysis makes it possible to find the regularities of the structural ordering of materials [3].

This paper aims to investigate the different scale substructural heterogeneities in the $\mathrm{Fe}_{77} \mathrm{Cu}_{1} \mathrm{Si}_{16} \mathrm{~B}_{6}$ alloy obtained by rapid quenching from the melt. Not only structural heterogeneities, but also their distribution, anisotropy, form factor, and their interconnection at different scale levels, are considered.

\section{Objects and research methods}

The objects of study were electron microscopic images of a 
spinning tape $\mathrm{Fe}_{77} \mathrm{Cu}_{1} \mathrm{Si}_{16} \mathrm{~B}_{6}$ obtained using a TITAN 300 transmission electron microscope (TEM), as well as images of the interfaces of a spinning tape (contact to the quenching disk and free from its influence) obtained using a scanning electron microscope Carl Zeiss Crossbeam 1540XB (SEM) [22-25]. The thickness of the samples is $\sim 20 \mu \mathrm{m}$; therefore, they were thinned for transmission electron microscopy. Bright-field images of a spinning ribbon were obtained from areas near the contact and free interfaces.

For all rapidly quenched alloys, a Fourier spectral analysis was carried out, including the following: integral frequency response (IFR), which allows numerically determining the range of inhomogeneities present in the studied structure and integrated spatial characteristic (ISC), based on which it is possible to judge the isotropy / anisotropy of the studied structure [26-30].

\section{Results and Discussion}

The conditions for obtaining alloys by rapid quenching from the liquid state are highly disbalanced due to significant cooling rates $\sim 10^{6} \mathrm{deg} / \mathrm{s}$, and $\operatorname{grad} \mathrm{T} \sim 100 \mathrm{deg} / \mu \mathrm{m}$, and other factors [1], which can lead to "freezing" of the short-range order.

The result of the performed X-ray structural analysis of the $\mathrm{Fe}_{77} \mathrm{Cu}_{1} \mathrm{Si}_{16} \mathrm{~B}_{6}$ sample using $\gamma$-radiation from $\mathrm{Cu}(\mathrm{K} \alpha, \lambda=1.54$ A) is shown in Fig.1. The calculation of the effective penetration depth of X-ray radiation into the alloy of the given composition is $(3-4)$ microns. Since the thickness of the spinning tape is $\sim 20 \mu \mathrm{m}$, the profiles of X-ray diffraction patterns from the contact and free interfaces of the sample were obtained, Fig.1. The X-ray diffraction patterns from the contact surface contain "crystalline" peaks, corresponding to $\alpha$-Fe solid solution with a chemical composition similar to the $\mathrm{Fe}_{3} \mathrm{Si}$, the size of the coherent scattering region is $1.5 \mathrm{~nm}$. The structure of the spinning ribbon on the free-side from the influence of the cooling cylinder is X-ray amorphous, Fig.1. Thus, the structure of the spinning $\mathrm{Fe}_{77} \mathrm{Cu}_{1} \mathrm{Si}_{16} \mathrm{~B}_{6}$ ribbon at the atomic level is represented by two components - amorphous and nanocrystalline. The obtained result suggests the existence of stratification, and the material can be considered as amorphous-nanocrystalline. The translational symmetry of the nanocrystalline phase and its absence in the amorphous matrix leads to distinctive physical properties of the material [1].

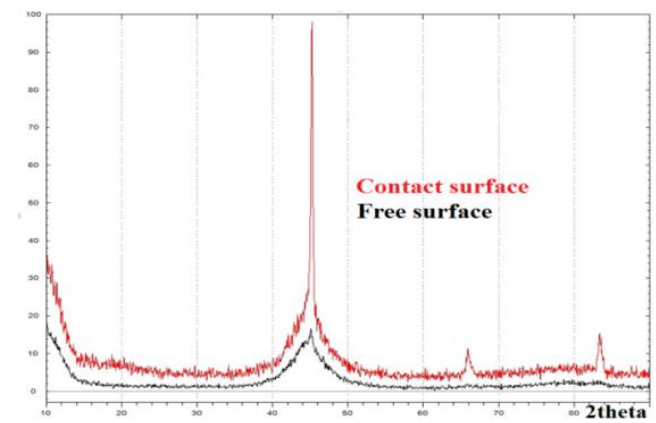

Fig.1. Profiles of X-ray diffraction patterns of a spinning ribbon $\mathrm{Fe}_{77} \mathrm{Cu}_{1} \mathrm{Si}_{16} \mathrm{~B}_{6}$, obtained from various interfaces.
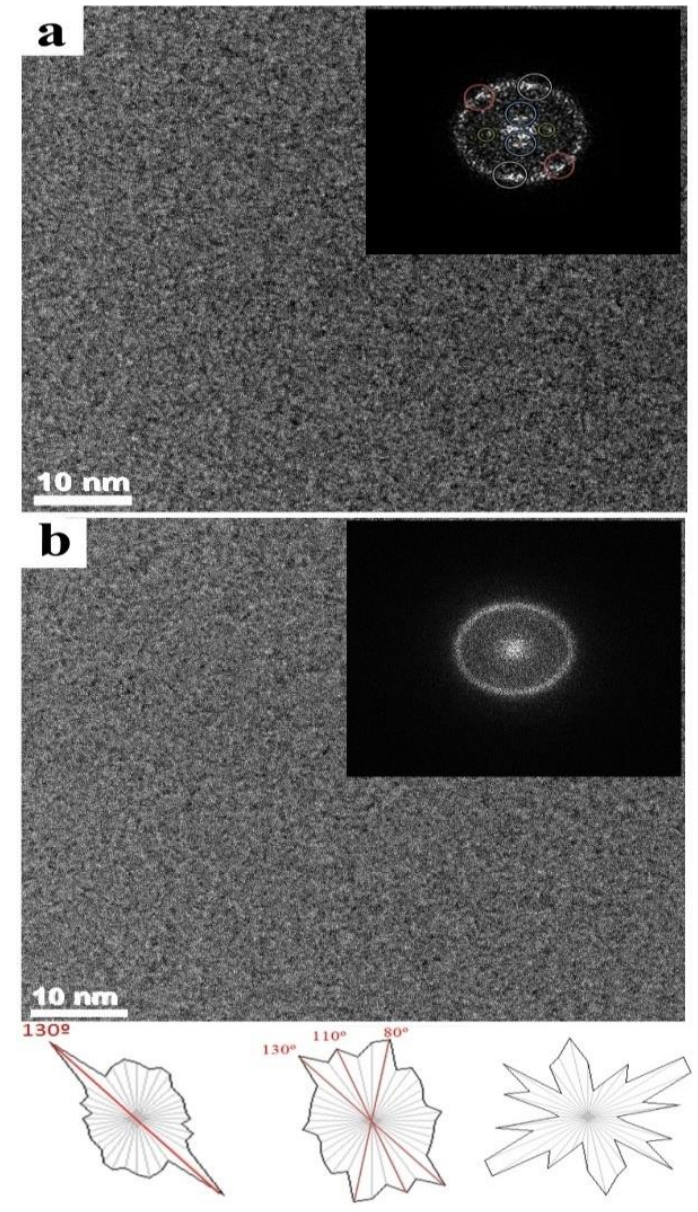

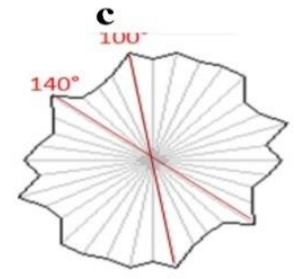

f d

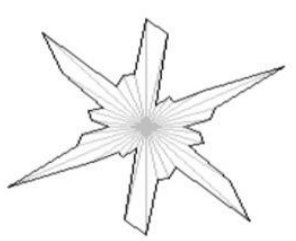

\section{$\mathbf{g}$}

Fig.2. Electron-microscopic image of the structure of a spinning ribbon $\mathrm{Fe}_{77} \mathrm{Cu}_{1} \mathrm{Si}_{16} \mathrm{~B}_{6}$ from area a) close to the contact surface; b) close to the free surface. Tabs show Fourier spectrum of image data and integrally spatial characteristic of periodicities: short wavelength $\lambda_{1}(\mathrm{c}, \mathrm{f})$; medium wavelength $\lambda_{2}$ (d); long wavelength $\lambda_{3}(\mathrm{e}, \mathrm{g})$ ranges.

Fig.2 shows electron microscopic TEM images of a spinning ribbon from regions near the contact (a) and free (b) surfaces, which demonstrate a homogeneous structure of the "salt-pepper" type with a clearly distinguishable period of inhomogeneities (wavelength) $\lambda_{1} \sim 0.2 \mathrm{~nm}$. A detailed analysis of the image makes it possible to visualize the combination of short-wavelength periodicities into larger irregularities with a period of $\sim 0.7 \mathrm{~nm}$ and more. However, visual analysis of the image does not allow us to assess the structure anisotropy.

Fig. 2 also shows the results of spectral analysis of the obtained TEM images of spinning ribbon from regions close to the interfaces and calculated by IFR and ISC.As a result of the spectral analysis of electron microscopic images of spinning ribbons, the following was obtained: the structure of 
the studied foils consists of inhomogeneities of various sizes, which correspond to certain frequency modes.

Three ranges of sizes of inhomogeneities were distinguished - short-wavelength, $\lambda_{1}=0.2 \mathrm{~nm}$; medium wavelength, $\lambda_{2}=$ $(0.4-0.7) \mathrm{nm}$; long wavelength, $\lambda_{3}=(1.36-2.27) \mathrm{nm}$.

An analysis of the ISC of the Fourier spectrum for the area near the contact surface showed a high degree of anisotropy of the structure for short wavelength and medium wavelength (Fig.2c,d). The mesoscale inhomogeneities with wavelength $\lambda^{2}$ are characterized by the existence of dispersion $\left(\sim 20^{\circ}\right)$ in their distribution. For the area near the free interface of the spinning ribbon, the distribution of inhomogeneities short range order is isotropic (the anisotropy coefficient $\varepsilon$ in the two directions is the same and is, $\varepsilon \sim 1.3$, Fig.2f).

Note that the form of the ISC for the periodicities of the long wavelength range $\left(\lambda_{3}\right)$ with the presence of several distinguished axes, Fig.2, do not make a significant contribution to the integral structure anisotropy, Table I.

Table I. Characteristics of nanostructured and morphological inhomogeneities.

\begin{tabular}{|l|c|c|c|c|}
\hline & \multicolumn{4}{|c|}{ SEM } \\
\hline & \multicolumn{2}{|c|}{ Contact surface } & \multicolumn{2}{c|}{ Free surface } \\
\hline & $\boldsymbol{\varepsilon}$ & $\boldsymbol{\Lambda}, \boldsymbol{\mu} \mathbf{m}$ & $\boldsymbol{\varepsilon}$ & $\boldsymbol{\Lambda}, \boldsymbol{\mu m}$ \\
\hline $\mathbf{1}$ & 1.75 & 2.8 & 1 & 3.2 \\
\hline $\mathbf{2}$ & 3 & 7.2 & 2 & 7.7 \\
\hline $\mathbf{3}$ & 3.6 & 20.3 & 3.3 & 18.1 \\
\hline \multicolumn{4}{|c|}{ TEM } \\
\hline \multicolumn{4}{|c|}{ The area close to } & \multicolumn{2}{|c|}{ The area close to } \\
the contact surface & the free surface \\
\hline & $\boldsymbol{\varepsilon}$ & $\boldsymbol{\lambda}, \mathbf{n m}$ & $\boldsymbol{\varepsilon}$ & $\boldsymbol{\lambda}, \mathbf{n m}$ \\
\hline $\mathbf{1}$ & 1.8 & 0.2 & 1.3 & 0.2 \\
\hline $\mathbf{2}$ & 1.34 & 0.6 & 1.14 & 0.5 \\
\hline $\mathbf{3}$ & 1 & 1.8 & 1 & 1.62 \\
\hline
\end{tabular}

Spinning ribbons $\mathrm{Fe}_{77} \mathrm{Cu}_{1} \mathrm{Si}_{16} \mathrm{~B}_{6}$ have two surfaces with different morphological. Fig. $3 \mathrm{a}$ shows an electron microscopic image of the contact surface of a spinning ribbon with a developed relief in the form of rolling strips and caverns of various sizes, shapes and orientations. An analysis of the image revealed a spectrum of inhomogeneities: from small ones with a period of $\sim 3 \mu \mathrm{m}$ (Fig.3a) aligned along the direction of rolling, to medium ones with a period of $\sim 10 \mu \mathrm{m}$, which have a fairly isotropic shape, and long-wavelengths with a period of $\sim 20 \mu \mathrm{m}$ in the form of non-cubic tetrahedra, which indicates the presence of anisotropy of the form. The free surface of the amorphous spinning ribbon is characterized by a more even tubercle relief, Fig.3b.

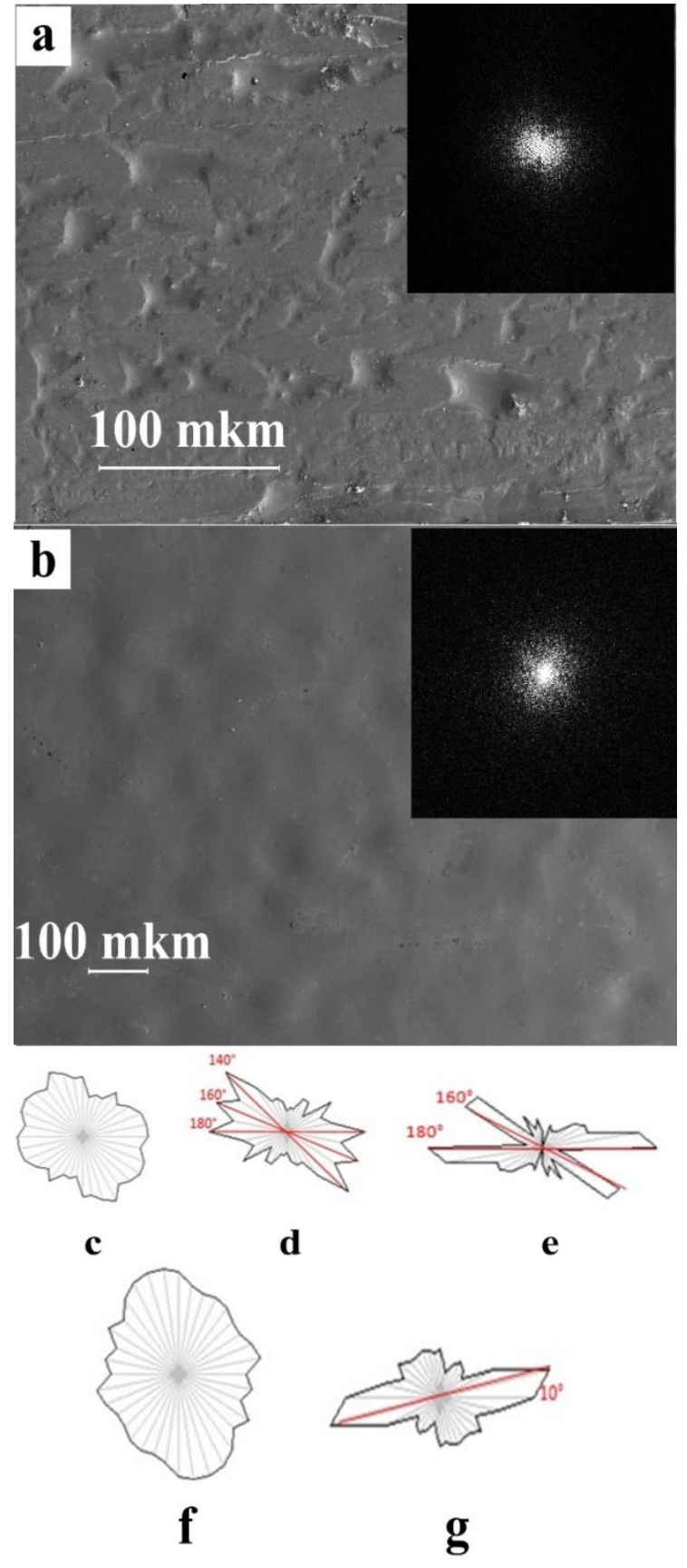

Fig. 3. Electron - microscopic image of a) contact surface; b) free surfacespinning ribbon $\mathrm{Fe}_{77} \mathrm{Cu}_{1} \mathrm{Si}_{16} \mathrm{~B}_{6}$. The tabs show the Fourier spectrum of the images data and the corresponding ISC and integrally spatial characteristic of periodicities: short wavelength $\Lambda_{1}$ (c, f); medium wavelength $\Lambda_{2}$ (d); long wavelength $\Lambda_{3}(\mathrm{e}, \mathrm{g})$ ranges.

Spectral Fourier analysis of electron microscopic images of the interfaces between rapidly quenched alloys, Fig.3a,b, revealed three ranges of sizes of inhomogeneities of the morphostructure of spinning ribbons: short-wavelength $\Lambda_{1}$, medium-wavelength $\Lambda_{2}$, long-wavelength $\Lambda_{3}$, and confirmed multiscale on the micrometer range, Table I.

Inhomogeneities of the short wavelength range $\Lambda_{1}$ of the contact surface are aligned along the rolling direction of the spinning ribbon and have an isotropic shape. The periodicities 
of the long wavelength range $\Lambda_{3}$ have an anisotropic form due to the induced shape anisotropy and the significant influence of spinning technology on them. Medium and long wave inhomogeneities are characterized by the presence of a dispersion of the anisotropy axis $\left(\sim 20^{\circ}\right)$, which is explained by the shape of the inhomogeneities (Fig.3d-e). It is shown that the integral anisotropy of the morphostructure of the spinning ribbon is determined by the periodicities of the long wavelength range, Table I.

Short wave inhomogeneities of the free surface are characterized by a lower anisotropy index $(\varepsilon=1)$ compared with the contact surface $(\varepsilon=1.75)$, Table I. The transition to high-size inhomogeneities leads to an increase in the anisotropy coefficient. The formation of the middle and long range order of inhomogeneities of the free surface is accompanied by a sharp increase in the anisotropy in their distribution and, by the value of $\varepsilon$, reaches the value of the anisotropy coefficient of the contact surface, Fig. $3 f-g$.

Thus, the behavior of inhomogeneities in the structure of spinning ribbons in the nanometer range differs from the morphological level, Fig.4. Anisotropy of this periodicity scale is formed by short wavelength inhomogeneities, $\lambda_{1}=0.2$ $\mathrm{nm}$, which have a maximum value of $\varepsilon$, both on the contact and on the free surfaces, Fig. $4 \mathrm{~b}$. The formation of mesoscale and long wavelength structures is accompanied by a decrease in the anisotropy coefficient, Fig.4b. This dependence pattern (Fig. 4b) is related to the elimination of uniaxial anisotropy for highdimensional homogeneities as shown above, Fig.2. The spectral analysis of the structure of rapidly quenched alloys at the morphological level detected opposite regularities, Fig.4a: the maximum anisotropy coefficient is a characteristic of the long-wave periodicities. The size reduction of inhomogeneities of the morphological structure of both spinning ribbon surfaces is accompanied by a decrease the anisotropy coefficient $\varepsilon$, Fig.4a.
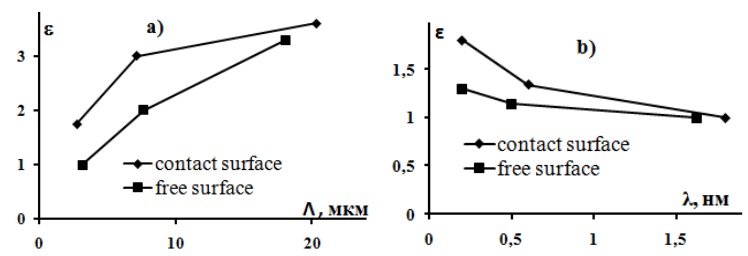

Fig. 4. The dependence of the anisotropy coefficient on the wavelength a) for the contact and free surface of the amorphous spinning ribbon (SEM); b) for the area close to the contact surface and the area close to the free surface (TEM).

After analyzing the results obtained, we can say that an inversion is observed between the structure and morphostructure of the rapidly quenched $\mathrm{Fe}_{77} \mathrm{Cu}_{1} \mathrm{Si}_{16} \mathrm{~B}_{6}$ ribbon in terms of the value of the anisotropy coefficient, which manifests itself in a change in the nature of anisotropy for different size ranges of inhomogeneities.

So, as a result of a spectral analysis of the structure of the $\mathrm{Fe}_{77} \mathrm{Cu}_{1} \mathrm{Si}_{16} \mathrm{~B}_{6}$, the presence of the drawn ranges is shown: short-wavelength, long-wavelength periodicities, and mesoscale range. Average values of the lengths revealed periodicities of the ribbon surfaces: $\Lambda_{1}-2.8 \mu \mathrm{m}$ and $3.2 \mu \mathrm{m}$, $\Lambda_{2}-7.2 \mu \mathrm{m}$ and $7.7 \mu \mathrm{m}, \Lambda_{3}-20.3 \mu \mathrm{m}$ and $18.1 \mu \mathrm{m}$, for the contact and free surfaces, respectively. Average values of the wavelengths of the revealed structural periodicities of the nanoscale: $\lambda_{1}-0.2 \mathrm{~nm}$ and $0.2 \mathrm{~nm}, \lambda_{2}-0.6 \mathrm{~nm}$ and $0.5 \mathrm{~nm}, \lambda_{3}-$ $1.8 \mathrm{~nm}$ and $1.62 \mathrm{~nm}$, for regions close to the contact and free surfaces, respectively.

The ratios $\lambda_{2} / \lambda_{1}$ and $\Lambda_{2} / \Lambda_{1}, \lambda_{3} / \lambda_{1}$ and $\Lambda_{3} / \Lambda_{1}$, were found for the wavelengths of the periodicities obtained by analyzing electron microscopic images from transmission and scanning electron microscopes, Table II.

It should be noted that these characteristics are close for the of both inhomogeneities levels: the ratios $\Lambda_{2} / \Lambda_{1}$ and $\Lambda_{3} / \Lambda_{1}$ have similar values both for the contact and free interfaces (morphological level) and for the regions of structures studied using a transmission electron microscope, $\lambda_{2} / \lambda_{1}$ and $\lambda_{3} / \lambda_{1}$. Moreover, the formation of medium and long-range order in an amorphous-nanocrystalline alloy goes on the principle of self-similar spatial structures characteristic of modulationunstable media [31-35], as evidenced by the correspondence of the wavelength ratios, Table II, for the nanometer and morphological ranges.

Table II. The ratios of the wavelengths ratios of the inhomogeneities of the nanometer range and the morphological level.

\begin{tabular}{|c|c|c|}
\hline \multicolumn{3}{|c|}{ SEM } \\
\hline $\begin{array}{c}\text { Wavelength } \\
\text { ratios }\end{array}$ & Contact surface & Free surface \\
\hline$\Lambda_{2} / \Lambda_{1}$ & 2.6 & 2.4 \\
\hline$\Lambda_{3} / \Lambda_{1}$ & 7.25 & 5.7 \\
\hline \multicolumn{3}{|c|}{ TEM } \\
\hline $\begin{array}{c}\text { Wavelength } \\
\text { ratios }\end{array}$ & $\begin{array}{c}\text { The area close to } \\
\text { the contact surface }\end{array}$ & $\begin{array}{c}\text { The area close to } \\
\text { the free surface }\end{array}$ \\
\hline$\lambda_{2} / \lambda_{1}$ & 3.0 & 2.5 \\
\hline$\lambda_{3} / \lambda_{1}$ & 9.0 & 8.1 \\
\hline
\end{tabular}

The next stage of the study of the amorphous spinning ribbon with the composition $\mathrm{Fe}_{77} \mathrm{Cu}_{1} \mathrm{Si}_{16} \mathrm{~B}_{6}$ is an analysis of structural changes in local areas. The image, Fig. 2, obtained in TEM, is a projection of the density of a fragment with a thickness of $(10-15) \mathrm{nm}$ : dark portions of the image are characterized by an increased density of the material compared to light. The Fourier spectrum, Fig. 2, obtained from an image measuring $(35 \times 35) \mathrm{nm}$, is isotropic with a diffuse halo located at a distance of $5 \mathrm{~nm}^{-1}(\sim 0.2 \mathrm{~nm})$ and reflects the nature of the structure in the integral representation, while the local regions are smaller and can have other characteristics.

To clarify the structural changes in the local areas, we selected regions of size $8.7 \mathrm{~nm} \times 8.7 \mathrm{~nm}$, which were shifted half a period in the horizontal direction along the image. Fourier spectra were obtained for all areas. Fig.5 shows electron microscopic images and their spectral characteristics for 2 random local regions of the $\mathrm{Fe}_{77} \mathrm{Cu}_{1} \mathrm{Si}_{16} \mathrm{~B}_{6}$ spinning ribbon near the contact surface. A visual analysis of electron microscopic images demonstrates differences in their structure. The obtained spectra, Fig.5b and Fig.5e, are isotropic, but bright reflections on the selected ring indicate the presence of local anisotropy. In order to introduce a quantitative measure of comparison, we use the methodology 
for calculating integral measures function of Lebesgue (IMFL) $[22-25,36]$. The starting point for the comparison procedure was the IFR obtained for seven local areas of the structure, an example of which is shown in Fig.5c and Fig.5f.

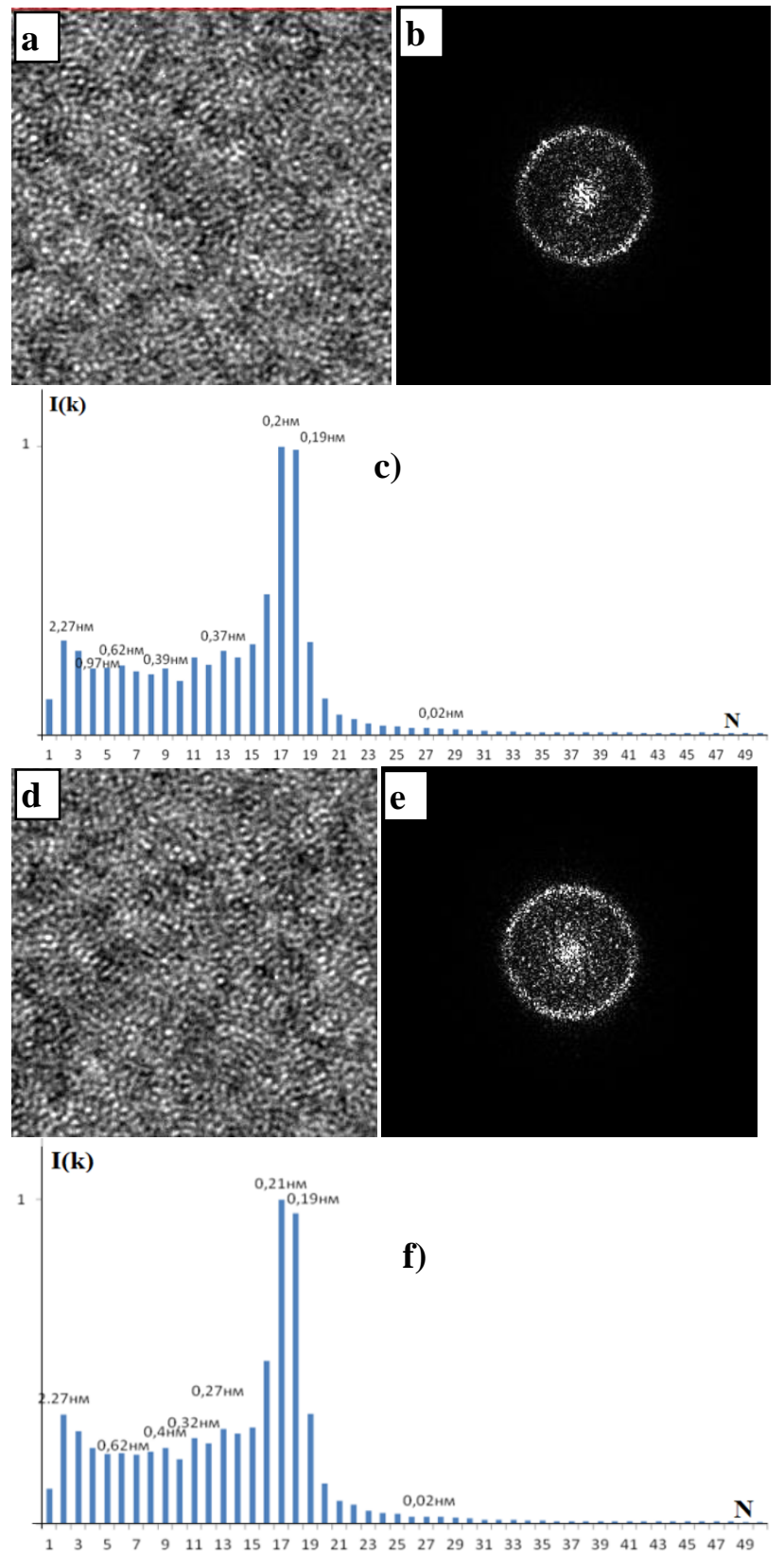

Fig.5. Electron microscopic images of the structure of a spinning ribbon $\mathrm{Fe}_{77} \mathrm{Cu}_{1} \mathrm{Si}_{16} \mathrm{~B}_{6}$ near the contact surface (8.7 $\mathrm{nm} \times 8.7 \mathrm{~nm}$ ) from two regions of the image shown in Fig. 1 (a) and the corresponding Fourier spectra (b,e) and IFR (c, f).

In information theory, divergent estimates of proximity and similarity of distributions are known. One of them is Kullback divergence (LivI) [23-25,29]. To construct it, the spectral estimate $\mathrm{I}(\mathrm{k})$ is chosen with linear IMFL corresponding to white noise.

Obtained dependence of the LivI on the area number is shown in Fig. 6a. As can be seen from the figure, the area at number 4 has the structure farthest from the structure of white noise. Thus, regions 1,2 , and 7 are the most structurally disordered. The methodology of our study in constructing the dependences (Fig. 6) assumes that region 2 consists of half of region 1 and half of region 3. Analysis of the results (Fig. 6a) shows that its characteristics are slightly different from region $1\left(\Delta \operatorname{Liv}_{1-2}=0.16\right)$ and highly different from region $3\left(\Delta \operatorname{Liv}_{1-3}\right.$ $=1.74)$. Therefore, the density projection changes at much smaller areas of the structure than the ones chosen for the study $(8.7 \mathrm{~nm} \times 8.7 \mathrm{~nm})$.

To confirm the result of a change in the local density characteristics of the structure of the spinning ribbon, another base was taken - the IFML of the entire image. That is, an analysis was made of the difference / similarity of the structure of local regions from integral ordering. This result is shown in Fig.6b.
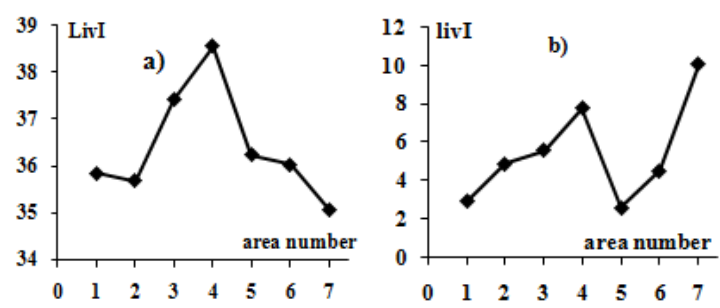

Fig.6. The Kullback divergence obtained from the IFML: a) the base is white noise, b) the base is the IFML from the frequency response of the entire image (Fig.2).

In addition to the already stated fact of a local change in the density projection, Fig.6a can carry additional information when it is jointly analyzed with Fig.6b. For example, the parameters of region 7, Fig. 6a, are closest to white noise, but are farthest from the integral IFML, Fig. 6b. The structure of region 4 is removed as from white noise, Fig. 6a, and from the average over the whole image, Fig.6b. Therefore, we can propose to consider cases of either of ordering, in the case of region 4, or disordering, in the case of region 7. Most likely, in terms of the projection of density, region 4 corresponds to a region with a higher density, and region 7 corresponds to a region with a lower density.

The above results were obtained for the TEM image from the structure located close to the contact surface of the spinning ribbon $\mathrm{Fe}_{77} \mathrm{Cu}_{1} \mathrm{Si}_{16} \mathrm{~B}_{6}$. In order to track the change in the projection of the structure density over the thickness of the ribbon, a similar procedure was performed for the image obtained from the structure located close to the free side of the ribbon, Fig. 2b. Analysis of the of IFML obtained for seven local regions of the structure near the free surface gave similar results.

The determined characteristic of structural heterogeneities of different spatial scales and their interconnection allow to consider the structure of a rapidly quenched $\mathrm{Fe}_{77} \mathrm{Cu}_{1} \mathrm{Si}_{16} \mathrm{~B}_{6}$ alloy as hierarchically arranged. By changing the production parameters of a rapidly quenched alloy from the melt it is possible to control the system of hierarchically connected structural heterogeneities, as well as the characteristics of the system and its subsystems. The adjustment of the characteristics of structural heterogeneities will make it 
possible to change the specified functional properties of the material.

\section{Conclusion}

Spectral analysis of electron microscopic images of a spinning ribbon $\mathrm{Fe}_{77} \mathrm{Cu}_{1} \mathrm{Si}_{16} \mathrm{~B}_{6}$ showed a complex nature of the structure, characterized by a wide range of inhomogeneities: three spatial ranges of sizes of inhomogeneitiesof nano - range and micro-level were identified: long-wave $\left(\lambda_{1}=1.7 \mathrm{~nm} ; \Lambda_{1}=\right.$ $19.2 \mu \mathrm{m})$; medium-wave $\left(\lambda_{2}=0.6 \mathrm{~nm} ; \Lambda_{2}=7.5 \mu \mathrm{m}\right)$, shortwavelength $\left(\lambda_{3}=0.2 \mathrm{~nm} ; \Lambda_{3}=2.8 \mu \mathrm{m}\right)$.

The anisotropy of the periodicities of the nanometer range is determined by short-wave inhomogeneities, $\lambda_{1}=0.2 \mathrm{~nm}$, which have a maximum value of $\varepsilon$, both on the contact surface and on the free one. The formation of mesoscale and longwavelength structures is accompanied by a decrease in the anisotropy coefficient. The integral anisotropy of the morphostructure of the spinning ribbon is determined by the inhomogeneities of the long-wavelength range.

A comparison of the spectral characteristics of the local areas of the structure of the rapidly quenched alloy revealed a different level of their formation. A change in the nature of anisotropy for the detected ranges of inhomogeneities of the nano and micro levels is reflected in the inversion of the anisotropy coefficient $\varepsilon$. The following result has been obtained: the formation of medium and long-range orders in an amorphous nanocrystalline alloy occurs on the principle of self-similar spatial structures characteristic of modulationunstable media.

Thus, the proposed TEM image processing technique allowed us to identify changes in the density projection that occur in the entire volume of the spinning ribbon $\mathrm{Fe}_{77} \mathrm{Cu}_{1} \mathrm{Si}_{16} \mathrm{~B}_{6}$. Most likely this is due to the wave process of heat removal during melt spinning.

The methods for describing multiscale structural heterogeneities in amorphous-nanocrystalline alloys have been developed, which allows to describe the structural state and determine its influence on the physicochemical and technical properties depending on the production of the alloys.

The results allow increasing the reliability in the investigation of the structure-property correlation of amorphous-nanocrystalline metal alloys obtained by rapid quenching from the melt.

\section{References}

[1] A. M. Glezer, N. A. Shurygina, Amorphousnanocrystalline alloys. CRC Press, 2017, 458p.

[2] F. Zhu, A. Hirata, P. Liu, S. Song, Y. Tian, J. Han, T. Fujita, M. Chen, Correlation between local structure order and spatial yeterogeneity in a metallic glass. Phys. Rev. Lett., 2017, vol. 119, pp. 215501.

[3] L. Tian, C.A. Volkert, Measuring structural heterogeneities in metallic glasses using transmission electron microscopy. Metals, 2018, vol.8, 1085, 14 p.

[4] L.L. Pang, A. Inoue, E.N. Zanaeva, F. Wang, A.I. Bazlov, Y. Han, F.L. Kong, S.L. Zhu, R.B. Shull, Nanocrystallization, good soft magnetic properties and ultrahigh mechanical strength for $\mathrm{Fe}_{82-85} \mathrm{~B}_{13-16} \mathrm{Si}_{1} \mathrm{Cu}_{1}$ amorphous alloys. J. of Alloys and Compounds, 2019, vol. 785, pp. 25-37.

[5] S. Cranford, Nature MADE: A Simple guide to biological design rules Matter, 2020, vol. 2, pp.782-785.

[6] F. Hou, Y. Yang, T. Luo, G. Wang, C. Fan, Z. Xie, Effect of Ni substitution to Fe on amorphous nanocrystalline soft magnetic alloy. Physica B, 2020, vol. 595, p.412293.

[7] D.V. Louzgune, Metallic glasses and their composites. Updated 2nd Edition, Published by Materials Research Forum LLC, PA 17551, USA, 2021, vol. 85, 340 p.

[8] V. Hasannaeimi, M. Sadeghilaridjani, S. Mukherjee, Electrochemical and corrosion behavior of metallic glasses. MDPI: Basel, Switzerland, 2021, 90 p.

[9] Z.X. Dou, Y.L. Li, K. Lv, T. Wang, F.S. Li, X.D. Hui, Improving the glass formation ability and magnetic properties by $\mathrm{Nb}$ in $\mathrm{Fe}-\mathrm{Si}-\mathrm{B}-\mathrm{P}-\mathrm{Cu}-\mathrm{Nb}$ nanocrystalline alloys. Materials Science and Engineering, 2021, vol. B 264, p. 114942.

[10] P.H. Gaskell, Medium-range structure in glasses and low-Q structure in neutron and X-ray scattering data. J. of Non-Crystalline Solids, 2005, vol. 351, pp. 1003-1013.

[11]H.W. Sheng, W.K. Luo, F. M. Alamgir, J.M. Bai, E. Ma, Atomic packing and short-to-mediumrange order in metallic glasses, Nature, 2006, vol. 439, pp. 419-425.

[12]H. Jinwoo, Z.H. Melgarejo, Y.E. Kalay, I. Kalay, M.J. Kramer, D.S. Stone, P.M. Voyles, Nanoscale structure and structural relaxation in $\mathrm{Zr}_{50} \mathrm{Cu}_{45} \mathrm{Al}_{5}$ bulk metallic glass. PRL, 2012, vol. 108, p. 195505.

[13]I.B. Kekalo, Processes of structural relaxation and physical properties of amorphous alloys. Publishing House "MISIS", 2014, vol. 1, 436 p.

[14] Y.Q. Cheng, E. Ma, Atomic-level structure and structureproperty relationship in metallic glasses. Progress in Materials Science, 2011, vol. 56, pp. 379-473.

[15]L. Hou, X. Fan, Q. Wang , W. Yang, B. Shen, Microstructure and soft-magnetic properties of FeCoPCCu nanocrystalline alloys. J. Mater. Sci. Technol., 2019, vol.35, Iss. 8, pp. 1655-1661.

[16]D. Azuma, N. Ito, M. Ohta, Recent progress in Fe-based amorphous and nanocrystalline soft magnetic materials. J. Magn. Magn. Mater., 2020, vol. 501, p. 166373.

[17]L. Hawelek, T. Warski, P. Wlodarczyk, M. Polak, P. Zackiewicz, W. Maziarz, A. Wojcik, M. SteczkowskaKempka, A. Kolano-Burian, The Structure and magnetic Properties of Rapidly Quenched $\mathrm{Fe}_{72} \mathrm{Ni}_{8} \mathrm{Nb}_{4} \mathrm{Si}_{2} \mathrm{~B}_{14}$. Alloy. Materials, 2021, vol. 14, №5, $10 \mathrm{p}$.

[18]F. Wang, A. Inoue, F.L. Kong, C.C. Zhao, J.Y. Zhang, S.L. Zhu, W.J. Botta, C.S. Kiminami, Y.P. Ivanov, A.L. Greer, Formation, thermal stability and mechanical properties of high-entropy $\left(\mathrm{Fe}_{0.25} \mathrm{Co}_{0.25} \mathrm{Ni}_{0.25} \mathrm{Cr}_{0.125} \mathrm{Mo}_{0.0625} \mathrm{Nb}_{0.0625}\right)_{100-\mathrm{x}} \mathrm{B}_{\mathrm{x}}(\mathrm{x}=7-14)$ amorphous alloys. J. of Alloys and Compounds, 2020, vol. 825, p. 153858.

[19]E.B. Modin, E.V. Pustovalov, A.N. Fedorets, A.V. Dubinets, B.N. Grudin, V.S. Plotnikov, S.S. Grabchikov, Atomic structure and crystallization processes of 
amorphous (Co, Ni)-P metallic alloy. J. of Alloys and Compounds, 2015, vol. 641, pp. 139-143.

[20]G. Herzer, Modern soft magnets: Amorphous and monocrystalline materials. ActaMaterialia, 2013, vol. 61, pp. 718-734.

[21] S. Kwon, S. Kim, H. Yim, Improvement of saturation magnetic flux density in $\mathrm{Fe}-\mathrm{Si}-\mathrm{B}-\mathrm{Nb}-\mathrm{Cu}$ nanocomposite alloys by magnetic field annealing. Current Applied Physics, 2020, vol. 20, pp. 37-42.

[22] L.A. Yudina, A.M. Frolov, N.I. Chukhry, V.V. Yudin, System method for complicated SEM images processing. Izvestiya Akademii Nauk. Ser. Fizicheskaya, 1998, vol. 62, pp. 455- 460.

[23] A.M. Frolov, G.S. Krainova, S.V. Dolzhikov, InformoDynamics functionals in the study of fields of anisotropy of a microrelief spinning tapes. Advanced Materials Research, 2014, vol.1025-1026, pp. 499-503.

[24]E.V. Pustovalov, A.N. Fedorets, V.V. Tkachev, V.S. Plotnikov, E.B. Modin, Regularities in the disordered atomic structure of rapidly quenched amorphous cobaltbased alloys. IEEE Conference Publications, 2017, pp. $142-144$.

[25]E.V. Pustovalov, E.B. Modin, A.M. Frolov, A.S. Kosovets, N.B. Kondrikov, N.F. Karpovich, S.A. Pyachin, S.V. Dolzhikov, G.S. Kraynova, V.S. Plotnikov, V.V. Tkachev, A.N. Fedorets, N.V. Ilin, Effect of the process conditions for the preparation of $\mathrm{CoNiFeSiB}$ amorphous alloys on their structure and properties. J. of Surface Investigation: X-ray, Synchrotron and Neutron Techniques, 2019, vol. 13, № 4, pp. 600-608.

[26]B.N. Grudin, V.S. Plotnikov, Processing and modeling of microscopic images. Vladivostok: Dalnauka, 2010, $350 \mathrm{p}$.

[27]B.N. Grudin, V.S. Plotnikov, E.V. Pustovalov, N.A. Smol'yaninov, S.V. Polishchuk, Simulation and analysis of images using spectral characteristics. Bulletin of the Russian Academy of Sciences: Physics, 2012, vol. 76, №9, pp. 1020-1024.

[28]B. N. Grudin, K.A. Petrov, V.S. Plotnikov, S.V. Polishchuk, E. B. Modin, Analyzing the fractal properties of a structure via microscopic images. Bulletin of the Russian Academy of Sciences: Physics, 2015, vol. 79, №11, pp. 1345-1349.

[29]A.M. Frolov, A.V. Ansovich, V.V. Tkachev, G.S. Kraynova, S.V. Dolzhikov, Surface morphology of spinning tapes $\mathrm{Fe}-(\mathrm{Cu}, \mathrm{Nb})-(\mathrm{Si}, \mathrm{B})$ with different content of metalloid. Key Engineering Materials, 2019, vol. 806, p. 124-129.

[30] A.M. Frolov, G.S. Krainova, S.V. Dolzhikov, Anisotropy of the structural inhomogeneities of rapidly quenched alloys. J. of Surface Investigation, 2018, vol. 12, p. 370376.

[31]M. Popescu, F. Sava, A. Lörinczi, Self-organization and anisotropy in amorphous chalcogenides. J. of NonCrystalline Solids, 2006, vol. 352, Iss. 9-20, pp. 15061509.

[32]I.E. Gracheva, V.A. Moshnikov, E.V. Maraeva, S.S. Karpova, O.A. Alexsandrova, N.I. Alekseyev, V.V. Kuznetsov, G. Olchowik, K.N. Semenov, A.V. Startseva,
A.V. Sitnikov, J.M. Olchowik, Nanostructured materials obtained under conditions of hierarchical self-assembly and modified by derivative forms of fullerenes. J. of Non-Crystalline Solids, 2012, vol. 358, Iss.2, pp. 433439.

[33]H.B. Ke, J.F. Zeng, C.T. Liu, Y. Yang, Structure heterogeneity in metallic glass: Modeling and Experiment J. Mater. Sci. Technol., 2014, vol. 30 Iss. 6, pp. 560-565.

[34]L. Tang, Z.J. Yang, T.Q. Wen, K.M. Ho, M.J. Kramer, C.Z. Wang, Short- and medium-range orders in $\mathrm{Al}_{90} \mathrm{~Tb}_{10}$ glass and their relation to the structures of competing crystalline phases. Acta Materialia, 2021, vol. 204, p. 116513.

[35]X. Tong, Y. Zhang, Y. Wang, X. Liang, K. Zhang, F. Zhang, Y. Cai, H. Ke, G. Wang, J. Shen, A. Makino, W. Wang, Structural origin of magnetic softening in a Febased amorphous alloy upon annealing. J. of Materials Science \& Technology, 2022, vol. 96, pp. 233-240.

[36] N.I. Chukhrii, V.V. Yudin, A.M. Frolov, L.A. Yudina, Correlation between quickquenched ribbon surfaces and atomic disordering in spinning processes. Surface Investigation. X-Ray, Synchrotron and Neutron Techniques, 2000, vol. 15, pp. 653-665.

\section{Author Contributions:}

Frolov A.M. - discuss the results, visualization, funding acquisition, writing - original draft, writing- review \& editing.

Ansovich A.V.- image processing.

Kraynova G.S.- methodology, writing - review \& editing, image processing

Tkachev V.V. - scanning electron microscopy investigation, data curation, visualization.

Dolzhikov, S.V. - discuss the results, data curation.

Plotnikov V.S. - discuss the results, data curation, supervision.

Ralin A.Yu. - transmission electron microscopy investigation, data curation.

Fedorets A.N. - transmission electron microscopy investigation, data curation, visualization.

\section{Sources of funding for research presented in a scientific article or scientific article itself}

This work was supported by the state task of the Ministry of Science and Higher Education of the Russian Federation under Grant № 0657-2020-0005.

The experimental results were obtained on the equipment of the Centre of Collective Usage of the Far Eastern Federal University, registration No. 200556 (Vladivostok).

Creative Commons Attribution License 4.0 (Attribution 4.0 International, CC BY 4.0)

This article is published under the terms of the Creative Commons Attribution License 4.0

https://creativecommons.org/licenses/by/4.0/deed.en_US 